\title{
A NEW METHOD FOR DETERMINING THE ACCURATE J-R CURVES OF STEELS
}

\author{
Hui Gao ${ }^{1}$, Chun-Qing Li $^{1}$, Weigang Wang ${ }^{1}$, Yanlin Wang ${ }^{1}$, and Bohua Zhang ${ }^{1}$ \\ ${ }^{1}$ RMIT University School of Engineering
}

January 11, 2021

\begin{abstract}
Unloading compliance (UC) method and normalization method (NM) are two of the most commonly used methods for determining the fracture toughness of materials. However, considerable differences often exist in the fracture toughness determined by these two methods, which solicits a new method to determine the fracture toughness accurately. In this paper, the compliance of crack length differences as measured by the crack length difference ratio Si is discovered, analysed and verified by experiments. Based on this compliance, a new accurate method, known as AJR, is developed and verified by test results. Factors that exhibit the advantages of the developed new AJR method are also investigated. It is found that the J-R curves determined by the new AJR method are more accurate than those determined by UC and NM. The new AJR method should be the first choice for steels with a small strain hardening ratio and low effective yield strength, and thicker CT specimens with shallower initial crack length. This is because the disagreement between UC and NM is unacceptably large. The developed new AJR method and the results presented in this paper can assist engineers and researchers to determine J-R curves and fracture toughness of steels more accurately and can contribute to the body of knowledge of fracture mechanics.
\end{abstract}

\section{Hosted file}

HG04_J-R curve_to FFEMS 09012021.pdf available at https://authorea.com/users/333335/articles/ 503464-a-new-method-for-determining-the-accurate-j-r-curves-of-steels 